\title{
Clinical significance of changes in the corrected QT interval in stress-induced cardiomyopathy
}

\author{
Jung-Hee Lee*, Jae-Sun Uhm*, Dong Geum Shin, Boyoung Joung, Hui-Nam Pak, Young-Guk Ko, \\ Geu-Ru Hong, and Moon-Hyoung Lee
}

Division of Cardiology, Department of Internal Medicine, Severance Cardiovascular Hospital, Yonsei University College of Medicine, Seoul, Korea

Received: July 31, 2015

Revised : August 24, 2015

Accepted: August 25, 2015

\section{Correspondence to}

Moon-Hyoung Lee, M.D.

Division of Cardiology,

Department of Internal Medicine,

Severance Cardiovascular

Hospital, Yonsei University

College of Medicine, 50-1

Yonsei-ro, Seodaemun-gu, Seoul

03722, Korea

Tel: +82-2-2228-8446

Fax: +82-2-2227-7732

E-mail: mhlee@yuhs.ac

*These authors contributed equally to this work.
Background/Aims: Although transient changes in the electrocardiogram (ECG) of patients with stress-induced cardiomyopathy (SCMP) are common, there are little data about ECG changes in patients with SCMP and the clinical implications of these variations.

Methods: We investigated a total of 128 patients (age, $63.2 \pm 15.4$ years; female, $60.9 \%)$ diagnosed with SCMP. We compared the ECGs taken after SCMP diagnosis and during the recovery phase to those taken before SCMP diagnosis under baseline conditions. All patients were divided into two groups according to corrected QT (QTc) interval changes: recovered QTc group (QTc in SCMP > QTc in recovery phase, $\mathrm{n}=77$ ) and nonrecovered QTc group (QTc in SCMP $\leq$ QTc in recovery phase, $\mathrm{n}=51$ ).

Results: In comparison of baseline, SCMP, and recovery phase, we found the mean heart rate ( $81.5 \pm 18.7,96.8 \pm 25.3$, and $83.0 \pm 19.4 / \mathrm{min}$, respectively; $p<0.001$ ), frequencies of ST segment elevation (0.0\%, 8.6\%, and 1.6\%, $p=0.004)$, ST segment depression (0.0\%, 6.3\%, and $1.6 \%, p=0.007)$, $\mathrm{T}$ wave inversion $(4.4 \%, 43.8 \%$, and $61.7 \%, p<0.001)$, and QTc $(447.4 \pm 35.3,488.9 \pm 67.1$, and $468.0 \pm 49.5, p<0.001)$ showed significant changes. In-hospital mortality (9.1\% vs. $25.5 \%, p=0.012$ ) and critical care $(54.5 \%$ vs. $72.5 \%, p=0.040$ ) occurred more frequently in the nonrecovered QTc group than in recovered QTc group.

Conclusions: The QTc can be prolonged in patients with SCMP. Short-term mortality was increased in patients where the QTc did not recover.

Keywords: Takotsubo cardiomyopathy; QTc interval; Electrocardiography; Mortality

\section{INTRODUCTION}

Stress-induced cardiomyopathy (SCMP), also known as apical ballooning syndrome or Takotsubo cardiomyopathy, is a cardiac syndrome characterized by transient left ventricular dysfunction that can mimic acute myocardial infarction (AMI) without obstructive coronary artery disease [1-3]. Almost two thirds of patients with SCMP experience an emotionally or physically stressful event $[3,4]$ which immediately precedes the cardiac event. SCMP is associated with regional wall motion abnormalities involving hypokinesia or akinesia of the mid and apical segments of the left ventricle, and these wall motion abnormalities typically resolve themselves within several days [5].

Previous reports have demonstrated that patients with SCMP have various abnormalities in their electrocardiogram (ECG) such as ST-segment elevation, T wave inversion, nonspecific ST-T abnormalities, and prolongation of the corrected QT (QTc) interval [6-8]. 
Critically, repolarization dispersion, such as the prolongation of QTc interval, was also seen in the acute and subacute phases of SCMP [9]. The QTc interval is longer in SCMP than in AMI, and typically resolves over 3 to 4 months $[9,10]$. The prolonged QTc intervals are at risk of ventricular arrhythmia, such as polymorphic ventricular tachycardia, in patients with acute phase of SCMP [11-13]. Although these transient electrocardiographic changes are common features in patients with SCMP, there are little data about the serial electrocardiographic changes in patients with SCMP during hospitalization. Furthermore, the clinical implications of the QTc interval prolongation in SCMP patients have not been fully investigated. Therefore, the aim of this study is to investigate the clinical significance of QTc interval changes in patients with SCMP.

\section{METHODS}

\section{Study design and patient selection}

We retrospectively analyzed the clinical data of 429 patients with a clinical diagnosis of SCMP between February 2009 and March 2014 at a single university hospital. We excluded patients with atypical ballooning types (transient hypokinesis, akinesis, or dyskinesis of the left ventricular mid segments without apical involvement), structural heart disease, and regional wall motional abnormalities that were not recovered. Our study population consisted of 128 patients who had typical apical ballooning with no lasting wall motion abnormalities. The Institutional Review Board of Severance Cardiovascular Hospital waived the requirement for informed consent for this retrospective analysis. Fig. 1 outlines the patient selection process.

\section{Diagnosis of SCMP}

All patients fulfilled the Mayo Clinic criteria for SCMP [1,14], and follow-up echocardiograms demonstrated complete resolution of the left ventricular dysfunction. We categorized the time course of SCMP as the baseline phase, the SCMP diagnosis phase, and the recovery phase. Each phase was defined by the following methods: (1) the baseline phase was defined as a clinical period before the presence of any cardiac dysfunction or abnormal cardiac events, (2) the SCMP diagnosis phase was defined

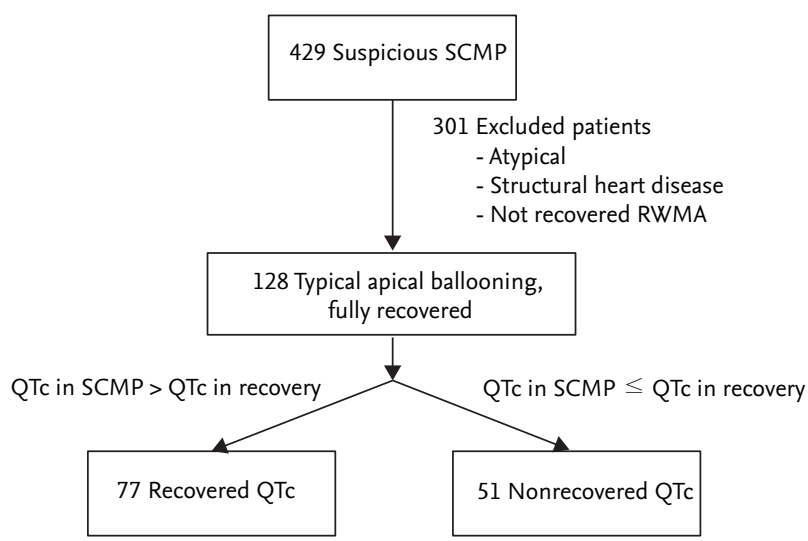

Figure 1. Study design and exclusion criteria. SCMP, stress-induced cardiomyopathy; RWMA, regional wall-motion abnormality; QTc, corrected QT.

as clinically diagnosed SCMP with the lowest ejection fraction (EF) assessed by echocardiogram, and (3) the recovery phase was defined as a clinical recovery with fully recovered left ventricular function assessed by echocardiogram. We evaluated ECG parameters during each of these phases. The wall motion score index (WMSI) was calculated by the average of all analyzed segment scores.

\section{Electrocardiogram analysis}

We evaluated all 128 study subjects' serial ECGs according to their serial echocardiographic findings, and we obtained the baseline ECG of 113 patients (88.3\%). Twelve-lead ECGs were recorded at a paper speed of 25 $\mathrm{mm} / \mathrm{sec}$ with a $10 \mathrm{~mm} / \mathrm{mV}$ amplification scale using the GE Marquette MUSE system (GE Medial System, Milwaukee, WI, USA). The high and low cut-off frequencies were 100 and $0.5 \mathrm{~Hz}$, respectively. The ECGs were simultaneously assessed by two investigators who were blinded of clinical characteristics, patients group, and in-hospital course.

We compared the serial ECG changes of each patient taking during each phase of their hospitalization: the baseline phase, the SCMP diagnosis phase, and the recovery phase. We acquired the following ECG parameters: heart rate, ST elevation $(>1 \mathrm{~mm}$ in at least two contiguous leads), ST depression (>1 $\mathrm{mm}$ in at least two contiguous leads), and $\mathrm{T}$ wave inversion (inverted $\mathrm{T}$ wave amplitude $>3 \mathrm{~mm}$ in at least three precordial or inferior leads). The QTc interval (corrected for heart rate according to Bazett's formula, QTc $=\mathrm{QT} / \sqrt{\mathrm{RR}}$ ) was automatically measured. We defined an abnormally prolonged 
QTc interval as $>450 \mathrm{~ms}$ in men and $>470 \mathrm{~ms}$ in women [15]. All patients were divided into two groups according to QTc interval changes: $(1)$ recovered QTc group (QTc in SCMP > QTc in recovery phase, $\mathrm{n}=77$ ), or (2) nonrecovered QTc group (QTc in SCMP $\leq$ QTc in recovery phase, $\mathrm{n}=51$ ). We excluded QTc prolongation that was induced by drugs and electrolyte imbalance.

\section{Study endpoints}

The primary endpoint was in-hospital all-cause mortality after study patients were diagnosed with SCMP. The secondary endpoints were incidences of critical care after SCMP diagnosis and the length of hospital stay. Critical care was defined as dependence on ventilator care or infusion of inotropes.

\section{Statistical analysis}

Statistical analysis was performed using SPSS version 20.0.0 (IBM Co., Armonk, NY, USA). Continuous variables were compared by an independent $t$ test after normality tests. Not normally distributed continuous variables were compared by the Mann-Whitney $U$ test. Data are expressed as number (\%) or as mean \pm standard deviation or median (interquartile range). Comparisons of categorical data were made using chi-square statistics or Fisher exact test. Changes in ECG parameters of each patient were compared with the use of generalized-estimating-equation models (for categorical outcomes) and repeated measures linear mixed-effects models (for continuous outcomes). In-hospital mortality was analyzed by Kaplan-Meier survival curves, and the differences between cumulative in-hospital mortality rates were compared with the log rank test. Multivariate logistic analysis was performed to determine the independent risk factors. The variables with a $p<0.05$ in the univariate analysis and traditional cardiac risk factors (age and gender) were included in the multivariate regression model. A $p<0.05$ was considered statistically significant.

\section{RESULTS}

\section{Baseline characteristics of the study population}

One hundred and twenty-eight patients were included in this study. The patients ranged in age from 15 to 90 years (mean age, 63.2 \pm 15.4 ), and were 60.9\% (78/128) female (Table 1). The most common trigger factor of SCMP was physical stress (89.1\%, 114/128). Pre-existing critical illness, such as sepsis, respiratory insufficiency, major noncardiac surgery, or cerebrovascular accident, were similar between two groups. The median time interval from SCMP diagnosis to recovery phase was 13.0 days (interquartile range [IQR], 7.0 to 32.0) and similar in the two groups (15.0 days [IQR, 7.0 to 38.0] in the recovered QTc group and 10.0 days [IQR, 7.0 to 23.0] in the nonrecovered QTc group, $p=0.199)$. Pharmacological treatment such as $\beta$-blocker or calcium channel blocker, potentially affecting the QTc interval and the arrhythmogenic risk, were similar between two groups.

\section{Serial changes of echocardiographic and electrocar- diographic parameters}

Baseline echocardiography of 51 patients and the baseline ECG of 113 patients were analyzed. Left ventricular EF was markedly decreased during the SCMP diagnosis phase compared to both baseline and the recovery phase $(65.5 \% \pm 6.9 \%$ vs. $36.1 \% \pm 11.4 \%$ vs. $63.6 \% \pm 7.7 \%$, respectively; $p<0.001$ ) (Table 2). Heart rate was also significantly elevated during the SCMP diagnosis phase $(81.5 \pm 18.7)$ min vs. $96.8 \pm 25.3 / \mathrm{min}$ vs. $83.0 \pm 19.4 / \mathrm{min}$, respectively; $p$ $<0.001$ ). ST elevation (0.0\% vs. $8.6 \%$ vs. $1.6 \%$, respectively; $p=0.004$ ) and ST depression (0.0\% vs. $6.2 \%$ vs. $1.6 \%$, respectively; $p=0.007$ ) were more frequently observed in the SCMP phase compared to either the baseline or recovery phases. However, $\mathrm{T}$ wave inversion was more frequently observed in the recovery phase than either the baseline or SCMP phase ( $4.4 \%$ vs. $43.8 \%$ vs. $61.7 \%$, respectively; $p<0.001)$. The QTc interval was significantly prolonged during the SCMP phase $(447.4 \pm 35.3 \mathrm{~ms}$ vs. $488.9 \pm 67.1 \mathrm{~ms}$ vs. $468.0 \pm 49.5 \mathrm{~ms}$, respectively; $p<0.001$ ), and returned to lengths observed in baseline after echocardiography showed recovery of left ventricular systolic function. The prevalence of QTc prolongation was also more frequently observed in the SCMP phase than the other phases ( $18.6 \%$ vs. $56.3 \%$ vs. $39.1 \%$, respectively; $p<$ o.001).

\section{Comparison of clinical, echocardiographic, and electrocardiographic characteristics between recov- ered vs. nonrecovered QTc group}

Even though the average QTc interval returned to baseline levels for all patients, the simultaneous recovery 
Table 1. Baseline characteristics

\begin{tabular}{|c|c|c|c|}
\hline Characteristic & Recovered QTc ( $\mathrm{n}=77)$ & Nonrecovered QTc $(\mathrm{n}=51)$ & $p$ value \\
\hline Age, yr & $63.3 \pm 14.8$ & $63.1 \pm 16.3$ & 0.958 \\
\hline Male sex & $29(37.7)$ & $21(41.2)$ & 0.690 \\
\hline Body surface area, $\mathrm{kg} / \mathrm{m}^{2}$ & $1.57 \pm 0.20$ & $1.58 \pm 0.19$ & 0.592 \\
\hline Hypertension & $41(53.2)$ & $19(37 \cdot 3)$ & 0.076 \\
\hline Diabetes & $18(23.4)$ & $16(31.4)$ & 0.316 \\
\hline Dyslipidemia & $2(2.6)$ & $1(2.0)$ & 0.816 \\
\hline Smoking & $17(22.1)$ & $8(15.7)$ & 0.372 \\
\hline History of cancer & $27(35 \cdot 1)$ & $18(35 \cdot 3)$ & 0.979 \\
\hline Chronic kidney disease & $6(7.8)$ & $4(7.8)$ & 0.992 \\
\hline Old cerebrovascular accident & $4(5.2)$ & $4(7.8)$ & 0.545 \\
\hline Trigger factor & & & 0.411 \\
\hline Emotional stress & $7(9.1)$ & $7(13.7)$ & \\
\hline Physical stress & $70(90.9)$ & $44(86.3)$ & \\
\hline Sepsis & $26(33.8)$ & $19(37 \cdot 3)$ & 0.386 \\
\hline Respiratory insufficiency & $20(26.0)$ & $17(33 \cdot 3)$ & 0.369 \\
\hline Major non-cardiac surgery & $12(15 \cdot 6)$ & $6(11.8)$ & 0.543 \\
\hline Cerebrovascular accident & $4(5.2)$ & 0 & 0.098 \\
\hline Systolic blood pressure, $\mathrm{mmHg}$ & $119 \cdot 3 \pm 17 \cdot 9$ & $117 \cdot 3 \pm 20.2$ & 0.559 \\
\hline Diastolic blood pressure, $\mathrm{mmHg}$ & $72.6 \pm 13.9$ & $71.8 \pm 14.1$ & 0.736 \\
\hline Heart rate, /min & $94 \cdot 5 \pm 24.0$ & $103.0 \pm 29.0$ & 0.078 \\
\hline Time interval SCMP diagnosis to recovery, day & $15(7-38)$ & $10(7-23)$ & 0.199 \\
\hline \multicolumn{4}{|l|}{ Ejection fraction, \% } \\
\hline At baseline & $64.8 \pm 7.6$ & $66.9 \pm 5.4$ & 0.306 \\
\hline At SCMP diagnosed & $37.8 \pm 11.3$ & $33.5 \pm 11.2$ & 0.034 \\
\hline At recovery & $63.6 \pm 8.0$ & $63.7 \pm 7 \cdot 3$ & 0.934 \\
\hline \multicolumn{4}{|l|}{ Wall motion score index } \\
\hline At SCMP diagnosed & $2.07 \pm 0.31$ & $2.09 \pm 0.32$ & 0.675 \\
\hline At recovery & $1.04 \pm 0.14$ & $1.04 \pm 0.13$ & 0.972 \\
\hline \multicolumn{4}{|l|}{ Medication } \\
\hline Calcium channel blocker & $6(7.8)$ & $1(2.0)$ & 0.155 \\
\hline$\beta$ Blocker & $14(18.2)$ & $6(11.8)$ & 0.328 \\
\hline \multicolumn{4}{|l|}{ ECG parameter } \\
\hline \multicolumn{4}{|l|}{ Heart rate, /min } \\
\hline At baseline & $80.8 \pm 18.5$ & $82.7 \pm 19.3$ & 0.610 \\
\hline At SCMP diagnosis & $92.3 \pm 22.5$ & $103 \cdot 5 \pm 27 \cdot 9$ & 0.014 \\
\hline At recovery & $82.6 \pm 20.4$ & $83.7 \pm 18.2$ & 0.775 \\
\hline \multicolumn{4}{|l|}{ ST elevation } \\
\hline At SCMP diagnosis & $8(10.4)$ & $3(5 \cdot 9)$ & 0.373 \\
\hline At recovery & $1(1.3)$ & $1(2.0)$ & 0.767 \\
\hline \multicolumn{4}{|l|}{ ST depression } \\
\hline At SCMP diagnosis & $5(6.5)$ & $3(5 \cdot 9)$ & 0.889 \\
\hline At recovery & $1(1.3)$ & $1(2.0)$ & 0.767 \\
\hline
\end{tabular}


Table 1. Continued

\begin{tabular}{|c|c|c|c|}
\hline Characteristic & Recovered QTc $(\mathrm{n}=77)$ & Nonrecovered QTc $(\mathrm{n}=51)$ & $p$ value \\
\hline \multicolumn{4}{|l|}{ T wave inversion } \\
\hline At SCMP diagnosis & $38(49.4)$ & $18(35 \cdot 3)$ & 0.117 \\
\hline At recovery & $49(63.6)$ & $30(58.8)$ & 0.583 \\
\hline \multicolumn{4}{|l|}{ QT prolongation } \\
\hline At SCMP diagnosis & $62(80.5)$ & $10(19.6)$ & $<0.001$ \\
\hline At recovery & $24(31.2)$ & $26(51.0)$ & 0.025 \\
\hline \multicolumn{4}{|l|}{ Corrected QT interval, ms } \\
\hline At baseline & $449 \cdot 9 \pm 38.0$ & $443.2 \pm 30.4$ & 0.322 \\
\hline At SCMP diagnosis & $516.9 \pm 45.9$ & $456.9 \pm 43.8$ & $<0.001$ \\
\hline At recovery & $456.3 \pm 45 \cdot 6$ & $485.6 \pm 50.3$ & 0.001 \\
\hline $\begin{array}{l}\text { Aggravated QTc prolongation (> } 500 \mathrm{~ms} \text { ) at SCMP } \\
\text { diagnosis }\end{array}$ & $49(63.6)$ & $9(17 \cdot 6)$ & $<0.001$ \\
\hline Aggravated QTc prolongation (> $500 \mathrm{~ms}$ ) at recovery & $15(19 \cdot 5)$ & $17(33 \cdot 3)$ & 0.076 \\
\hline \multicolumn{4}{|l|}{ Laboratory findings } \\
\hline WBC, count $/ \mu \mathrm{L}$ & $11,691 \pm 7,259$ & $11,174 \pm 7,017$ & 0.696 \\
\hline Hemoglobin, g/dL & $10.8 \pm 2.9$ & $10.8 \pm 1.7$ & 0.994 \\
\hline CPK, IU/L & $427 \cdot 3 \pm 789.6$ & $332.4 \pm 589.3$ & 0.486 \\
\hline CK-MB, ng/mL & $14.2 \pm 24 \cdot 3$ & $19.2 \pm 40.0$ & 0.387 \\
\hline Troponin-T, ng/mL & $0.4 \pm 0.7$ & $1.2 \pm 5.6$ & 0.314 \\
\hline $\mathrm{CRP}, \mathrm{mg} / \mathrm{L}$ & $98.3 \pm 100.5$ & $100.6 \pm 98.6$ & 0.904 \\
\hline NT-pro BNP, pg/mL & $9,55^{8} \pm 12,585$ & $6,863 \pm 9,072$ & 0.364 \\
\hline
\end{tabular}

Values are presented as mean $\pm \mathrm{SD}$, number (\%), or median (interquartile range).

QTc, corrected QT; SCMP, stress-induced cardiomyopathy; WBC, white blood cell; CPK, creatine phosphokinase; CK-MB, creatine kinase MB; CRP, C-reactive protein; NT-pro BNP, N-terminal of the prohormone brain natriuretic peptide.

Table 2. Serial changes of echocardiographic and electrocardiogram parameters during baseline, stress-induced cardiomyopathy, and recovery

\begin{tabular}{|c|c|c|c|c|c|c|}
\hline Echocardiographic parameter & Baseline $(n=51)$ & $\operatorname{SCMP}(n=128)$ & Recovery $(n=128)$ & $p$ value & $p$ value $^{a}$ & $p$ value $^{b}$ \\
\hline Ejection fraction, \% & $65.5 \pm 6.9$ & $36.1 \pm 11.4$ & $63.6 \pm 7.7$ & $<0.001$ & $<0.001$ & $<0.001$ \\
\hline Wall motion score index & $1.0 \pm 0.0$ & $2.1 \pm 0.3$ & $1.0 \pm 0.1$ & $<0.001$ & $<0.001$ & $<0.001$ \\
\hline ECG parameters & 113 & 128 & 128 & & & \\
\hline Heart rate, /min & $81.5 \pm 18.7$ & $96.8 \pm 25 \cdot 3$ & $83.0 \pm 19.4$ & $<0.001$ & $<0.001$ & $<0.001$ \\
\hline Sinus rhythm & $104(92.0)$ & $111(86.7)$ & $118(92.2)$ & 0.038 & 0.030 & 0.034 \\
\hline Atrial fibrillation & $8(7 \cdot 1)$ & $12(9.4)$ & $8(6.3)$ & 0.256 & 0.191 & 0.099 \\
\hline Ventricular premature complex & $1(0.9)$ & $3(2.3)$ & $2(1.6)$ & 0.658 & 0.367 & 0.654 \\
\hline ST segment elevation & 0 & $11(8.6)$ & $2(1.6)$ & 0.004 & $<0.001$ & 0.004 \\
\hline ST segment depression & $\mathrm{O}$ & $8(6.3)$ & $2(1.6)$ & 0.007 & 0.002 & 0.108 \\
\hline T wave inversion & $5(4 \cdot 4)$ & $56(43.8)$ & $79(61.7)$ & $<0.001$ & $<0.001$ & $<0.001$ \\
\hline QT interval, ms & $390.8 \pm 48.1$ & $396.8 \pm 82.1$ & $407.3 \pm 66.7$ & 0.027 & 0.904 & 0.366 \\
\hline Corrected QT interval, ms & $447 \cdot 4 \pm 35 \cdot 3$ & $488.9 \pm 67.1$ & $468.0 \pm 49.5$ & $<0.001$ & $<0.001$ & 0.004 \\
\hline Prolonged QTc & $21(18.6)$ & $72(56.3)$ & $50(39.1)$ & $<0.001$ & $<0.001$ & 0.004 \\
\hline
\end{tabular}

Values are presented as mean \pm SD or number (\%).

SCMP, stress-induced cardiomyopathy; ECG, electrocardiogram; QTc, corrected QT.

${ }^{\text {a}}$ Baseline vs. SCMP.

${ }^{\mathrm{b}} \mathrm{SCMP}$ vs. recovery. 

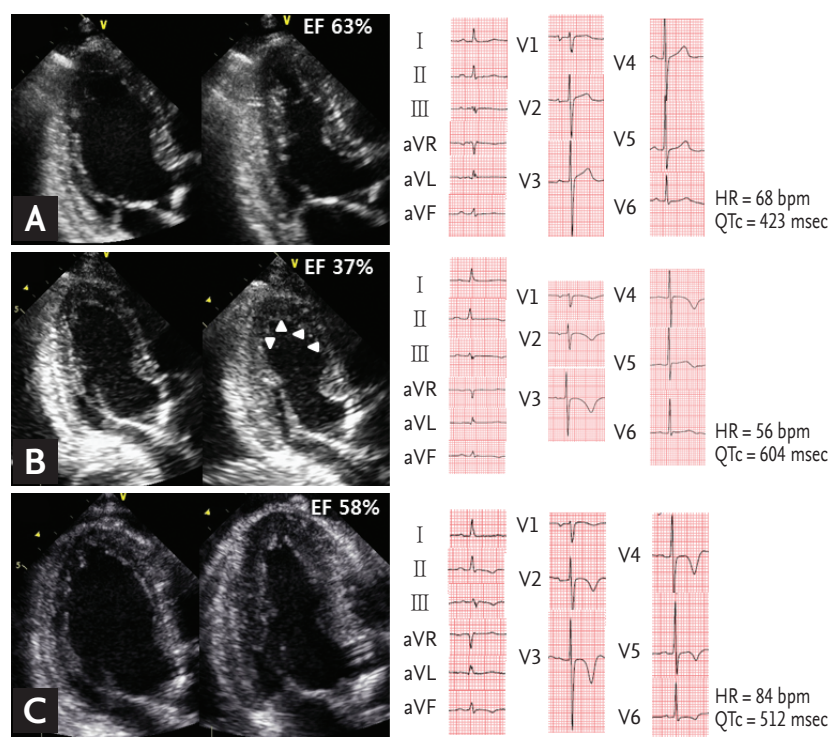

Figure 2. The echocardiography and electrocardiogram of 64-years old male presenting stress-induced cardiomyopathy. He was categorized as the recovered corrected QT (QTc) group. (A) The baseline phase, (B) the stress-induced cardiomyopathy diagnosis phase, (C) the recovery phase. Arrowheads indicated the typical apical ballooning. EF, ejection fraction; $\mathrm{HR}$, heart rate.

of left ventricular systolic function with an attenuated QTc interval was not observed in all patients. Of all the patients in this study, 77 patients were categorized into the recovered QTc group (QTc in SCMP > QTc in recovery phase) (Fig. 2), and 51 patients were categorized into the nonrecovered QTc group (QTc in SCMP $\leq$ QTc in recovery phase) (Fig. 3). The baseline characteristics of these two groups were not significantly different (Table 1). Echocardiography showed that there were no differences in left ventricular EF at baseline or recovery between the two groups. However, the nonrecovered QTc group showed a lower EF compared to the recovered QTc group $(33.5 \% \pm 11.2 \%$ and $37.8 \% \pm 11.3 \%$, respectively; $p=0.034$ ) during the SCMP diagnosis phase. The WMSI was $2.08 \pm 0.31$ when study patients were diagnosed with SCMP, and there was no statistical difference between two groups, even if their left ventricular systolic function fully recovered.

The heart rate during the SCMP diagnosis phase of the nonrecovered QTc group was faster than that of the recovered QTc group (103.5 $\pm 27.9 / \mathrm{min}$ vs. $92.3 \pm 22.5 / \mathrm{min}$, $p=0.014)$, but the frequencies of ST elevation, ST depression, and $\mathrm{T}$ wave inversion showed no significant differences between the two groups. There was a signifi-

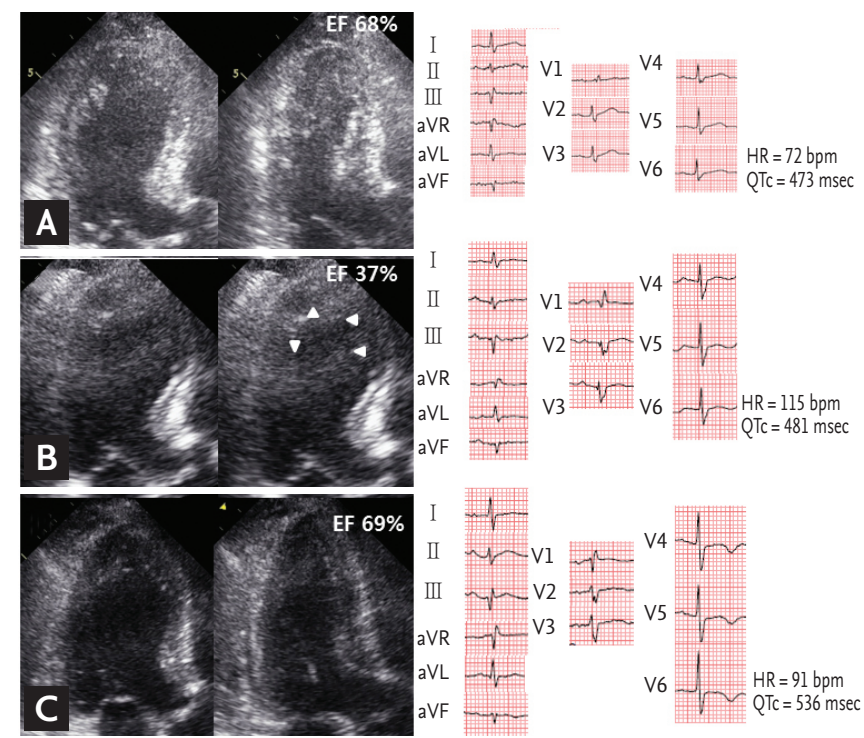

Figure 3. The echocardiography and electrocardiogram of 89 -years old female presenting stress-induced cardiomyopathy. She was categorized as the nonrecovered corrected QT (QTc) group. (A) The baseline phase, (B) the stress-induced cardiomyopathy diagnosis phase, $(\mathrm{C})$ the recovery phase. Arrowheads indicated the typical apical ballooning. EF, ejection fraction; HR, heart rate.

cant difference in QTc during both the SCMP diagnosis and recovery phases when comparing the recovered and nonrecovered QTc group $(522.9 \pm 53.2 \mathrm{~ms}$ vs. $437.7 \pm 51.9$ $\mathrm{ms}, p<0.001$ at SCMP diagnosis; $456.3 \pm 45.6 \mathrm{~ms}$ vs. 485.6 $\pm 50.3 \mathrm{~ms}, p=0.001$ at recovery).

\section{Nonrecovered QTc interval is predictor for in-hospi- tal mortality}

There was no difference between two groups with regard to peak creatinine kinase-MB values and the length of hospital stay. In-hospital all-cause mortality occurred more frequently in the nonrecovered QTc group than in the recovered QTc group (25.5\% vs. 9.1\%, $p=0.012$ ) (Table 3, Fig. 4). Severe sepsis and respiratory failure were the major causes of death in nonrecovered QTc group (17.6\% vs. $5.2 \%, p=0.022$ ). The incidence of critical care was also more frequently observed during hospitalization in the nonrecovered QTc group than in the recovered QTc group (72.5\% vs. 54.5\%, p = 0.040) (Table 3). In univariate logistic regression analysis, underlying cancer (odds ratio [OR], 2.66o; 95\% confidence interval [CI], 1.008 to $7.017 ; p=0.048$ ) (Table 4 ), heart rate at SCMP (OR, 1.036; 95\% CI, 1.009 to 1.064) and nonrecovered QTc (OR, 3.421; $95 \%$ CI, 1.258 to 9.301) were associated with in- 
Table 3. Clinical outcomes

\begin{tabular}{|c|c|c|c|}
\hline Variable & Recovered QTc (n = 77) & Nonrecovered QTc $(\mathrm{n}=51)$ & $p$ value \\
\hline Peak CK-MB, ng/mL & $7 \cdot 4(3 \cdot 3-13.8)$ & $7.6(3.6-18.1)$ & 0.593 \\
\hline Hospital stay, day & $21(9-37)$ & $19(9-30)$ & 0.800 \\
\hline Critical care ${ }^{a}$ & $42(54 \cdot 5)$ & $37(72.5)$ & 0.040 \\
\hline In-hospital mortality & $7(9.1)$ & $13(25 \cdot 5)$ & 0.012 \\
\hline Ventricular arrhythmia & O & $2(3.9)$ & 0.080 \\
\hline Infection or respiratory failure & $4(5.2)$ & $9(17 \cdot 6)$ & 0.022 \\
\hline Cancer at terminal stage & $3(3.9)$ & o & 0.154 \\
\hline
\end{tabular}

Values are presented as median (interquartile range) or number (\%).

QTc, corrected QT; CK-MB, creatine kinase MB.

${ }^{\mathrm{a}} \mathrm{Critical}$ care was defined as ventilator care or infusion of inotropics.

creased risk of in-hospital mortality. Using multivariate logistic regression analysis, the significant determinant for in-hospital mortality was nonrecovered QTc (OR, 3.811; $95 \%$ CI, 1.307 to 11.111; $p=0.014$ ), along with heart rate as a predictor of in-hospital mortality (OR, 1.039; $95 \% \mathrm{CI}, 1.009$ to $1.070 ; p=0.011$ ).

All 20 in-hospital mortality cases occurred upon physical stress, and these patients had near complete recovery of left ventricular EF. Two of these patients had developed sudden cardiac arrest, and it was highly suspected that these patients suffered from ventricular tachycardia. These patients were also were categorized into the nonrecovered QTc group.

\section{DISCUSSION}

The major findings of this study are that (1) ECG parameters of ST segment changes, or QTc interval could indicate whether left ventricular dysfunction present at the time of SCMP diagnosis was recovered or not, and (2) patients whose QTc interval did not recover to baseline levels during the SCMP recovery phase had a higher rate of in-hospital mortality or critical care.

Transient changes in the ECG of patients with SCMP are commonly observed. A previous study reported the time course of these ECG changes and demonstrated a deepened $\mathrm{T}$ wave inversion and a doubling of the QTC interval in SCMP patients [10]. Our findings are consistent with this report. We extended these findings by matching ECG parameters with the time course of echocardiographic changes when possible. This allowed

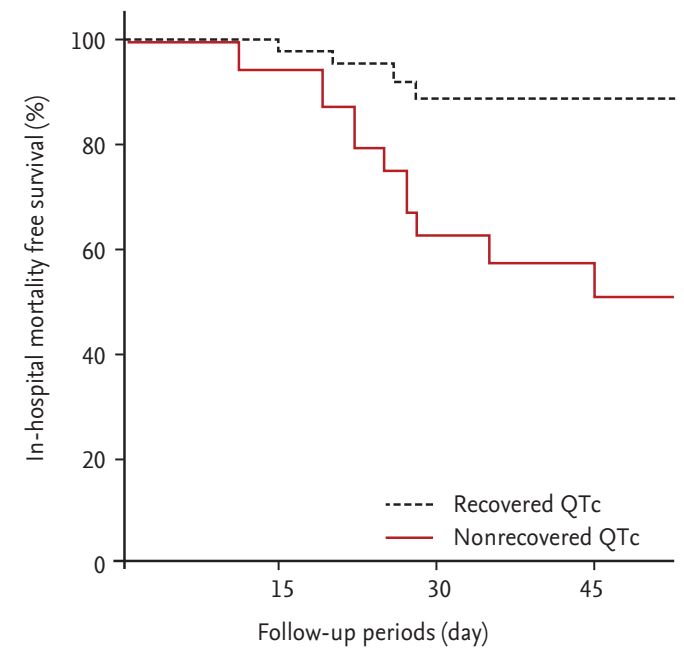

Recovered QTc (n) 77 Nonrecovered QTc(n) 51

50

25

12

13

Figure 4. Kaplan-Meier for cumulative in-hospital mortality incidence (median follow-up duration, 19.5 days; $p=0.012$ by log-rank). QTc, corrected QT.

us to demonstrate that both ST elevation or depression normalization and $\mathrm{T}$ wave inversions co-varied with recovery from left ventricular dysfunction. Systolic dysfunction and the regional wall motion abnormalities usually return to normal within several weeks $[5,6,16]$. Currently, the recovery of left ventricular dysfunction is typically evaluated by echocardiography only, but our data suggests that ECG parameters such as ST change, $\mathrm{T}$ wave properties, or the QTc interval may also be useful to help determine if recovery from SCMP has occurred.

In our study, when left ventricular EF had recovered 
Table 4. Logistic-regression model for in-hospital mortality

\begin{tabular}{|c|c|c|c|c|}
\hline \multirow{2}{*}{ Variable } & \multicolumn{2}{|c|}{ Univariate analysis } & \multicolumn{2}{|c|}{ Multivariate analysis } \\
\hline & OR $(95 \% \mathrm{CI})$ & $p$ value & OR $(95 \% \mathrm{CI})$ & $p$ value \\
\hline Age, /yr & $1.013(0.979-1.047)$ & 0.461 & $1.006(0.970-1.044)$ & 0.753 \\
\hline Male sex & $1.700(0.651-4.438)$ & 0.278 & $1.709(0.592-4.936)$ & 0.322 \\
\hline Diabetes & $1.224(0.429-3.495)$ & 0.705 & & \\
\hline Hypertension & $0.429(0.153-1.198)$ & 0.106 & & \\
\hline Dyslipidemia & $2.789(0.241-32.313)$ & 0.412 & & \\
\hline Smoking & $2.008(0.684-5.894)$ & 0.205 & & \\
\hline Underlying cancer & $2.660(1.008-7.017)$ & 0.048 & $2.301(0.783-6.762)$ & 0.130 \\
\hline Underlying critical illness ${ }^{\mathrm{a}}$ & $5.141\left(0.653-40.45^{8}\right)$ & 0.120 & & \\
\hline Chronic kidney disease & $0.579(0.069-4.840)$ & 0.614 & & \\
\hline Ejection fraction (\%) at SCMP & $0.989(0.947-1.032)$ & 0.598 & & \\
\hline Heart rate $(/ \mathrm{min})$ at SCMP & $1.036(1.009-1.064)$ & 0.008 & $1.039(1.009-1.070)$ & 0.011 \\
\hline Aggravated QTc prolongation (> $500 \mathrm{~ms}$ ) at SCMP & $0.985(0.378-2.570)$ & 0.976 & & \\
\hline Aggravated QTc prolongation (> $500 \mathrm{~ms}$ ) at recovery & $0.714(0.220-2.318)$ & 0.575 & & \\
\hline ST elevation at SCMP & $0.516(0.062-4.269)$ & 0.539 & & \\
\hline ST depression at SCMP & $0.759(0.088-6.531)$ & 0.802 & & \\
\hline T inversion at SCMP & $1.348(0.518-3.506)$ & 0.541 & & \\
\hline Nonrecovered QTc & $3.421(1.258-9.301)$ & 0.016 & $3.811(1.307-11.111)$ & 0.014 \\
\hline
\end{tabular}

OR, odds ratio; CI, confidence interval; SCMP, stress-induced cardiomyopathy; QTc, corrected QT.

${ }^{\mathrm{a}}$ Critical illness was defined as a consist of sepsis, respiratory insufficiency, major non-cardiac surgery, or cerebrovascular accident.

in patients with SCMP, we found that 77/128 patients (60.2\%) presented with a recovered QTc interval, and 51/128 patients (39.8\%) presented with a still prolonged QTc interval. Although most of the clinical parameters were similar between the two groups, patients in the nonrecovered QTc group showed a lower left ventricular EF at the time of SCMP diagnosis and were more likely to be associated with in-hospital mortality. While a history of cancer was one of the risk factors for in-hospital mortality in univariate analysis, only nonrecovered QTc interval was an independent risk factor for in-hos pital mortality. Previous report showed that pre-existing critical illness, such as sepsis, respiratory insufficiency, major noncardiac surgery, or cerebrovascular accident, were related with higher in-hospital mortality rate [17]. Our study suggested that pre-existing critical illness was not sufficient to significantly increased in-hospital mortality. We may also consider electrocardiographic changes including QTc in patient with SCMP.

The predictive value of QT dispersion for adverse outcomes has been evaluated in populations with myocar- dial infarction, and QT dispersion was demonstrated as a predictive factor for malignant ventricular arrhythmia $[18,19]$. Previous studies also demonstrated that the QT interval might be influenced by the extent of myocardial damage in patients with chronic myocardial infarction [20]. A previous report demonstrated that SCMP is rarely associated with malignant ventricular arrhythmia, even though both a significantly long QT interval and repolarization dispersion were observed [9]. However, in 2005 Denney et al. [11] reported a case of SCMP with QT interval prolongation in a young man who developed torsade de pointes and it now can carry a small but significant lethal risk for adverse outcomes, including cardiac mortality [21]. Cho et al. [13] also reported a case of SCMP presenting as VT as an initial manifestation. In our study, only two of the 20 in-hospital mortality cases (10\%) had developed sudden cardiac arrest. Both these patients suffered from malignant ventricular arrhythmia at the time of death, suggesting that this might be one of the mechanisms responsible for the observed in-hospital mortality. Furthermore, severe form of septic shock 
and respiratory failure were the major causes of death in nonrecovered QTc group. It is also hypothesized that severe extensive inflammatory reaction accompanying catecholamine surge with myocardial dysfunction can be related with continued repolarization abnormality.

Although overall in-hospital mortality from SCMP is very low, in the subgroup of patients where a physical stressor was present, such as illness or major surgery, prognosis was worse [4]. A recent study divided patients with SCMP into three groups according to ECG abnormalities at presentation such as ST elevation, $\mathrm{T}$ wave inversion, or non-specific ST-T abnormalities, but these abnormalities did not correlate with clinical outcomes [8]. That study categorized three groups according to ECG findings present only at SCMP presentation, but our study analyzed serial ECG changes during hospitalization, and demonstrated that a nonrecovered QTc interval was correlated with adverse outcomes. Our study is the first study using serial ECG and echocardiographic changes, and our data is the first risk predicting model for patients diagnosed with SCMP. In other words, by analyzing several ECG parameters of patient with SCMP, we can predict the patient's clinical phase of SCMP and in-hospital outcomes.

This study had several limitations. First, it was a retrospective study based on medical records. Further prospective studies are required to examine the significance of QTc interval changes. Second, we included only patients with SCMP who showed fully recovered left ventricular EF by echocardiography during the recovery phase. This may have potential for selection bias. Therefore, these results are only applicable to patients with follow-up echocardiography. Third, not all study populations performed coronary angiography for the purpose of excluding left ventricular dysfunction due to coronary artery disease. In our study, only 46 patients (35.9\%) underwent coronary angiography. However, we only included typical apical ballooning that fulfilled with Mayo Clinic criteria for SCMP and fully recovered, and we excluded patients with possible ischemic heart disease. Fourth, not all baseline ECGs or echocardiographic data of each patient were available. However, by using linear mixed-effects models and generalized-estimation-equation models, we can analyze all serial ECGs and echocardiographic data of each patient.

Some ECG parameters can be a surrogate marker of the clinical outcomes of patients presenting with SCMP. Even in SCMP patients who had complete recovery of left ventricular systolic function, a continued QTc prolongation was an independent risk factor for in-hospital mortality.

\section{KEY MESSAGE}

1. Electrocardiogram parameters, such as ST segment changes, or corrected QT (QTc) interval, could be useful to determine whether left ventricular dysfunction was recovered or not.

2. A continued QTc prolongation can be associated with increased risk of in-hospital mortality in patients with stress-induced cardiomyopathy.

\section{Conflict of interest}

No potential conflict of interest relevant to this article was reported.

\section{Acknowledgments}

The authors would like to acknowledge the contribution of the members of the Department of Research Affairs, Biostatistics Collaboration Unit, Yonsei University College of Medicine: Yun Ho Roh, M.S, Bo Gyoung Ma, M.S.

\section{REFERENCES}

1. Prasad A, Lerman A, Rihal CS. Apical ballooning syndrome (Tako-Tsubo or stress cardiomyopathy): a mimic of acute myocardial infarction. Am Heart J 2008;155:408417 .

2. Dote K, Sato H, Tateishi H, Uchida T, Ishihara M. Myocardial stunning due to simultaneous multivessel coronary spasms: a review of 5 cases. J Cardiol 1991;21:203-214.

3. Gianni M, Dentali F, Grandi AM, Sumner G, Hiralal R, Lonn E. Apical ballooning syndrome or takotsubo cardiomyopathy: a systematic review. Eur Heart J 2006;27:15231529.

4. Elesber AA, Prasad A, Lennon RJ, Wright RS, Lerman A, Rihal CS. Four-year recurrence rate and prognosis of the apical ballooning syndrome. J Am Coll Cardiol 2007;50:448-452. 
5. Tsuchihashi K, Ueshima K, Uchida T, et al. Transient left ventricular apical ballooning without coronary artery stenosis: anovel heart syndrome mimicking acute myocardial infarction: angina pectoris-myocardial infarction investigations in Japan. J Am Coll Cardiol 2001;38:11-18.

6. Sharkey SW, Lesser JR, Zenovich AG, et al. Acute and reversible cardiomyopathy provoked by stress in women from the United States. Circulation 2005;111:472-479.

7. Bybee KA, Kara T, Prasad A, et al. Systematic review: transient left ventricular apical ballooning: a syndrome that mimics ST-segment elevation myocardial infarction. Ann Intern Med 2004;141:858-865.

8. Dib C, Asirvatham S, Elesber A, Rihal C, Friedman P, Prasad A. Clinical correlates and prognostic significance of electrocardiographic abnormalities in apical ballooning syndrome (Takotsubo/stress-induced cardiomyopathy). Am Heart J 2009;157:933-938.

9. Matsuoka K, Okubo S, Fujii E, et al. Evaluation of the arrhythmogenecity of stress-induced "Takotsubo cardiomyopathy" from the time course of the 12-lead surface electrocardiogram. Am J Cardiol 2003;92:230-233.

10. Kurisu S, Inoue I, Kawagoe T, et al. Time course of electrocardiographic changes in patients with tako-tsubo syndrome: comparison with acute myocardial infarction with minimal enzymatic release. Circ J 2004;68:77-81.

11. Denney SD, Lakkireddy DR, Khan IA. Long QT syndrome and torsade de pointes in transient left ventricular apical ballooning syndrome. Int J Cardiol 2005;100:499-501.

12. Singh K, Carson K, Hibbert B, Le May M. Natural history of cardiac arrest in patients with takotsubo cardiomyopathy. Am J Cardiol 2015;115:1466-1472.

13. Cho SC, Kim W, Park CS, et al. Stress-induced cardiomyopathy presenting as ventricular tachycardia. Korean J Intern Med 2012;27:107-110.

14. Scantlebury DC, Prasad A. Diagnosis of Takotsubo cardiomyopathy. Circ J 2014;78:2129-2139.

15. Straus SM, Kors JA, De Bruin ML, et al. Prolonged QTc interval and risk of sudden cardiac death in a population of older adults. J Am Coll Cardiol 2006;47:362-367.

16. Wittstein IS, Thiemann DR, Lima JA, et al. Neurohumoral features of myocardial stunning due to sudden emotional stress. N Engl J Med 2005;352:539-548.

17. Brinjikji W, El-Sayed AM, Salka S. In-hospital mortality among patients with takotsubo cardiomyopathy: a study of the National Inpatient Sample 2008 to 2009. Am Heart J 2012;164:215-221.

18. Glancy JM, Garratt CJ, Woods KL, de Bono DP. QT dispersion and mortality after myocardial infarction. Lancet 1995;345:945-948.

19.Zareba W, Moss AJ, le Cessie S. Dispersion of ventricular repolarization and arrhythmic cardiac death in coronary artery disease. Am J Cardiol 1994;74:550-553.

2o.Hashimoto N, Musha H, Ozawa A, et al. Relationship between infarction location and size to QT dispersion in patients with chronic myocardial infarction. Jpn Heart J 2002;43:455-461.

21. Sharkey SW, Maron BJ. Epidemiology and clinical profile of Takotsubo cardiomyopathy. Circ J 2014;78:2119-2128. 